\title{
Comparison of different microstructure scaffolds for tissue regeneration
}

\author{
Andžela Šešok ${ }^{1}$, Deividas Mizeras², Algirdas Vaclovas Valiulis ${ }^{3}$, Julius Griškevičius ${ }^{4}$, \\ Mangirdas Malinauskas ${ }^{5}$ \\ 1,2,3,4 Vilnius Gediminas Technical University, Lithuania \\ ${ }^{5}$ Vilnius University, Lithuania \\ E-mails: ${ }^{1}$ andzela.sesok@vgtu.lt (corresponding author), ${ }^{2}$ deividas.mizeras@vgtu.lt, \\ ${ }^{3}$ algirdas.valiulis@vgtu.lt, ${ }^{4}$ julius.griskevicius@vgtu.lt, ${ }^{5}$ mangirdas.malinauskas@ff.vu.lt \\ (Received 9 March 2016; accepted 22 April 2016)
}

\begin{abstract}
In this work we aim to determine the mechanical properties of 3D printed PLA objects having various orientation woodpile microarchitectures. In this work we chose three different 3D microarchitectures: woodpile BCC (each layer consists of parallel logs which are rotated $90 \mathrm{deg}$ every next layer), woodpile FCC (every layer is additionally shifted half of the period in respect to the previous parallel log layer) and a rotating woodpile $60 \mathrm{deg}$ (each layer is rotated $60 \mathrm{deg}$ in respect to the previous one). Compressive and bending tests were carried out TIRAtest2300 universal testing machine. We found that $60 \mathrm{deg}$ rotating woodpile geometry had the highest values which was approximately 3 times than the BCC or FCC log arrangements. Thus we prove that employing low-cost equipment and applying the same raw material one can create objects of desired rigidity.
\end{abstract}

Keywords: scaffolds, polylactic acid (PLA), microstructure.

\section{Introduction}

Three-dimensional (3D) scaffolds are used with the intention to repair, replace, or regenerate injured tissues and organs (for example bone, cartilage, skin, liver, heart, lung and other). Ideally, a tissue engineering scaffold should be biocompatible, biodegradable, highly porous and interconnected, and mechanically reliable. Scaffolds mechanical properties are one of the main aspects to take into account for the development of 3D matrices: stiffness and strength, microstructure changes (resulting from the mechanical deformation of the surface), orientation. The exact control of these factors have a significant influence on the mechanical properties $[1,2]$.

In this work we applied polylactic acid (PLA) material and used it as supplied by the manufacturer. PLA is one of the most widely used synthetic polymers in biomedical products for drug delivery, orthopaedics, sutures, and scaffolds for tissue engineering [3].

$3 \mathrm{D}$ printing based on fused filament fabrication (FFF) is emerging as a tool for rapid prototyping as well as additive manufacturing [4]. It offers production of complex shaped microobjects with high feature spatial definition. Mechanical properties of such printed objects will depend dramatically on the applied material, geometry and filling factor.

In this work we aim to determine the mechanical properties of 3D printed PLA objects having various orientation woodpile microarchitectures.

\section{Methods}

Structures were created out of polylactic acid (PLA) using fabrication speed of $15 \mathrm{~mm} / \mathrm{s}$ and layer height $-50 \mu \mathrm{m}$. This allowed us to reach good compromise between fabrication throughput and quality of finished structure. We employ FFF 3D printer "Ultimaker" for the manufacturing of woodpile structures having $1.2 \mathrm{~mm}$ lattice period and $~ 50 \%$ fill factor.

Mechanical tests for plastics must be carried strictly on equal terms as set out in the relevant standards. In this work we analysed mechanical testing standards for plastics $[5,6]$ were formed 
3D microporous structures of PLA mechanical properties experimental testing methodology adapted to such samples. Most of the existing research attention was focused on the assessment of behavioural scaffolds during compression. Also equally important of scaffolds is tensile and bending properties. Are used standardized mechanical testing methods. Compressive and bending tests were carried out TIRAtest2300 universal testing machine (Germany) with the computer power and displacement measurement system. The methods and equipment have been chosen for their versatility, accuracy and accessibility. Machine used to test the samples suitable for testing of polymers. Load measurement error is less than $\pm 1 \%$. Step displacement measuring device accuracy $\pm 0.5 \%$. The specimens for compressive tests are prepared according to ISO 604 recommendations. The measurements of the specimens and measurement process correspond to this standard recommendation. Initial scaffolds height was measured by a micrometre $0.01 \mathrm{~mm}$. The specimens were placed between two standard solid clamping plates in test machine. The testing speed was $1 \mathrm{~mm} / \mathrm{min}$. Mechanical compression test was repeated for all specimens and recorded graphs of the load/displacement.

Bending tests are performed according to ISO 178. The measurements of the specimens and measurement process correspond to this standard recommendation. Initial samples height was measured by a micrometre $0.01 \mathrm{~mm}$. The distance between supports is $64 \mathrm{~mm}$. The supports are rounded, rounded radius is $5 \mathrm{~mm}$. The testing speed was $1 \mathrm{~mm} / \mathrm{min}$.

In this work we chose three different 3D microarchitectures: woodpile $\mathrm{BCC}$ (each layer consists of parallel logs which are rotated 90 deg every next layer), woodpile FCC (every layer is additionally shifted half of the period in respect to the previous parallel log layer) and a rotating woodpile $60 \mathrm{deg}$ (each layer is rotated $60 \mathrm{deg}$ in respect to the previous one). The 3D microarchitectures are shown in Fig. 1.

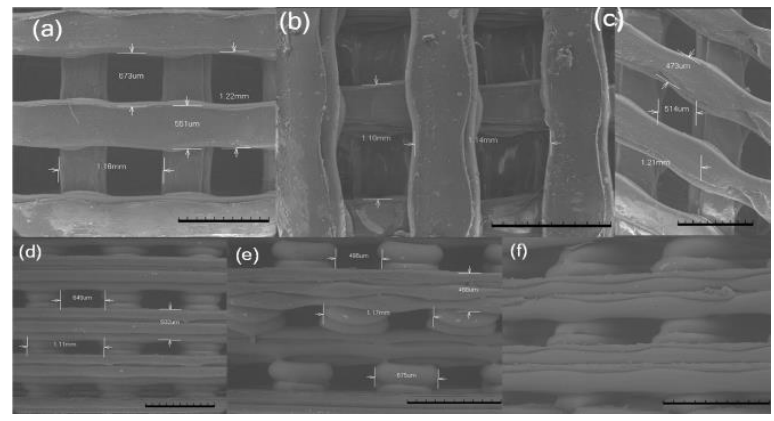

Fig. 1. SEM micrographs of 3D microarchitectures: woodpile (BCC), woodpile (FCC) and a rotating woodpile (60 deg); top and oblique views, (a-c) and (d-f), respectively

\section{Results}

Compression test results. Stress-strain curves are shown in Fig. 2. The maximum compressive strength limit, the elastic modulus and stiffness were characterized specimens of 60 deg geometry. This shows that printing orientation (3D architecture) has an influence on the mechanical properties.

Bending test results. Stress/deflection curve is presented in Fig. 3. 


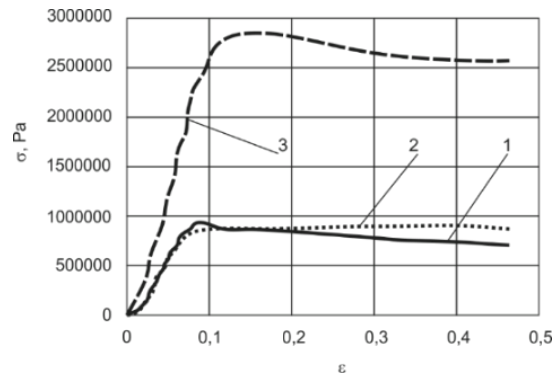

Fig. 2. Stress-strain curves: 1 - woodpile BCC; 2 - woodpile FCC; 3 - woodpile 60 deg

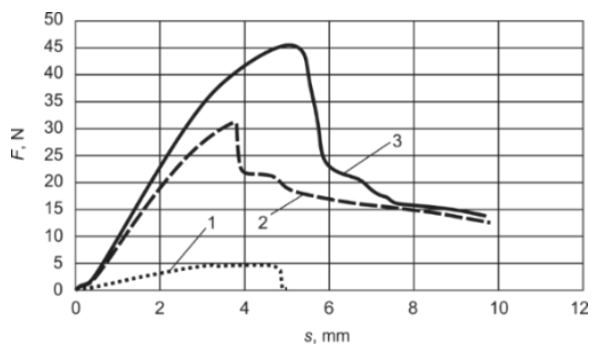

Fig. 3. A graph of stress/deflection curve: 1 - woodpile BCC; 2 - woodpile FCC; 3 - woodpile $60 \mathrm{deg}$

The lowest values of strength limit by bending had woodpile BCC. 60 deg rotating woodpile geometry had the highest values.

\section{Conclusions}

In this work we 3D printed objects having internal woodpile geometries and experimentally measured stress-strain and load/displacement curves of such specimens. 3 different geometries varying woodpile arrangement were examined by means of compression and bending enabling to experimentally determine elastic modulus, strength limit and stiffness. Within the limitation of the study we show that micro-architecture (variation of log orientation in respect to each other) can significantly modify the mechanical properties. We found that 60 deg rotating woodpile geometry had the highest values which was approximately 3 times than the BCC or FCC log arrangements. Thus we prove that employing low-cost equipment and applying the same raw material one can create objects of desired rigidity. By means of additive manufacturing one can produce objects with specific micro-architectures which allows exploitation the structural advantages of stretching and compression constructions as well as size dependent strengthening effects. Future work is focused on modeling mechanical properties of 3D printed objects dependence on internal microarchitecture and experimentally measuring more specimens in order to evaluate the repeatability.

\section{References}

[1] Malinauskas, M., et al. 2015. Tailoring bulk mechanical properties of 3D printed objects of polylactic acid varying internal micro-architecture, in Proceedings of SPIE Vol. 9505: Quantum Optics and Quantum Information Transfer and Processing, April 13, 2015, Prague, Czech Republic. Bellingham: SPIE, 95050P. http://dx.doi.org/10.1117/12.2178515

[2] Wolfe, P. S., et al. 2011. Natural and synthetic scaffolds, in Tissue engineering. Ed. by N. Pallua, C. V. Suschek. Berlin Heidelberg: Springer, 41-67. http://dx.doi.org/10.1007/978-3-642-02824-3_3

[3] Nair, L.; Laurencin, S. I. 2007. Biodegradable polymers as biomaterials, Progress in Polymer Science 32(8-9): 762-798. http://dx.doi.org/10.1016/j.progpolymsci.2007.05.017

[4] Malinauskas, M., et al. 2014. 3D microporous scaffolds manufactured via combination of fused filament fabrication and direct laser writing ablation, Micromachines 5(4): 839-858. http://dx.doi.org/10.3390/mi5040839

[5] EN ISO 178:2003 Plastics - determination of flexural properties. Brussels, 2003. 28 p.

[6] EN ISO 604:2002 Plastics - determination of compressive properties. Brussels, 2002. $21 \mathrm{p}$. 